\section{Retention of gene products in syncytial spermatids promotes non-Mendelian inheritance as revealed by the $t$ complex responder}

\author{
Nathalie Véron, ${ }^{1,2}$ Hermann Bauer, ${ }^{1}$ \\ Andrea Y. Weiße, ${ }^{3,6}$ Gerhild Lüder, ${ }^{4}$ \\ Martin Werber, ${ }^{1,5}$ and Bernhard G. Herrmann ${ }^{1,5,7}$ \\ ${ }^{1}$ Department of Developmental Genetics, Max-Planck-Institute \\ for Molecular Genetics, 14195 Berlin, Germany; ${ }^{2}$ Faculty of \\ Biology, Free University Berlin, 14195 Berlin, Germany; \\ ${ }^{3}$ Department of Mathematics and Computer Science, \\ Biocomputing Group, Free University Berlin, 14195 Berlin, \\ Germany; ${ }^{4}$ Electron Microscopy Group, Max-Planck-Institute \\ for molecular Genetics, 14195 Berlin, Germany; ${ }^{5}$ Institute for \\ medical Genetics-CBF, Charité-University Medicine Berlin, \\ 12203 Berlin, Germany
}

The $t$ complex responder (Tcr) encoded by the mouse $t$ haplotype is able to cause phenotypic differences between $t$ and + sperm derived from $t /+$ males, leading to non-Mendelian inheritance. This capability of Tcr contradicts the concept of phenotypic equivalence proposed for sperm cells, which develop in a syncytium and actively share gene products. By analyzing a Tcr minigene in hemizygous transgenic mice, we show that Tcr gene products are post-meiotically expressed and are retained in the haploid sperm cells. The wild-type allele of Tcr, sperm motility kinase-1 (Smok1), behaves in the same manner, suggesting that Tcr/Smok reveal a common mechanism prone to evolve non-Mendelian inheritance in mammals.

Supplemental material is available at http://www.genesdev.org.

Received August 14, 2009; revised version accepted October $12,2009$.

Diploid organisms transmit homologous chromosomes at an equal (the Mendelian) ratio to their offspring. However, several exceptions have been observed all across the animal kingdom and have puzzled geneticists for decades (Hurst and Werren 2001). In mammals, the mouse $t$ haplotype is a paradigm for non-Mendelian inheritance (Schimenti 2000; Lyon 2003). This variant of chromosome 17 is transmitted at excess rates (up to $99 \%$ ) from $t /+$ heterozygous males to their offspring (Chesley and Dunn 1936). This phenomenon, termed transmission ratio distortion (TRD), is caused by the action of several $t$ complex

[Keywords: Mouse; $t$ haplotype; non-Mendelian inheritance; transmission ratio distortion; spermatogenesis; motility]

${ }^{6}$ Present address: Hamilton Institute, National University of Ireland, Maynooth, County Kildare, Ireland

${ }^{7}$ Corresponding author.

E-MAIL herrmann@molgen.mpg.de; FAX 49-30-8413-1229.

Article is online at http://www.genesdev.org/cgi/doi/10.1101/gad.553009. distorters (Tcd1-4), which additively enhance the transmission rate of the $t$ complex responder (Tcr) (Lyon 1984). On the physiological level, TRD has been attributed to alteration of motility parameters in wild-type sperm derived from $t /+$ males (Katz et al. 1979; Olds-Clarke and Johnson 1993).

Tcr (gene symbol Smok1 ${ }^{T c r}$, herein abbreviated Tcr) was identified as a dominant-negative allele of the sperm motility kinase-1 (Smok1) (Herrmann et al. 1999). This finding suggested that Tcds are involved in signaling pathways controlling sperm motility via Smok1. Indeed, molecular cloning of Tcd1a and Tcd2 has identified regulators of Rho small G-proteins, Tagap1 and Fgd2, linking TRD to Rho signaling pathways (Bauer et al. 2005, 2007).

The current model of TRD suggests that Tcds additively contribute to deregulation of two Rho signaling cascades that control wild-type Smok1 protein (Bauer et al. 2005, 2007). The activating pathway is up-regulated, while the repressing pathway is down-regulated, by the Tcd gene products. The resulting hyperactivation of Smok1 compromises the flagellar motility of all $(t$ and +$)$ sperm derived from a $t /+$ male. The dominant-negative action of Tcr is able to specifically rescue $t$ sperm from this "poisonous" effect of the Tcds via an unknown mechanism (Herrmann et al. 1999). Thus, $t /+$ males produce two phenotypically distinct sperm populations, $t$ sperm and + sperm, which differ in sperm motility, leading to an advantage of $t$ sperm in fertilizing the egg cells (Supplemental Fig. 1).

This phenotypic difference between $t$ sperm and + sperm contradicts the proposed phenotypic equivalence between gametes, enabled by sharing of gene products between meiotic partners (Braun et al. 1989; Ventela et al. 2003). The latter has been demonstrated with a reporter transgene expressed exclusively in post-meiotic germ cells in hemizygous males (Braun et al. 1989). More recent experiments demonstrated microtubuli-dependent active transport of ribonucleoprotein (RNP) particles between haploid spermatids (Ventela et al. 2003). Thus, the question remained as to how Tcr causes gametic inequality.

\section{Results and Discussion}

We asked whether Tcr was able to escape the mechanism of gene product sharing between germ cells. To answer this question, we isolated the Tcr promoter and constructed a Tcr minigene that included a Myc epitope tag at the $\mathrm{N}$ terminus of the Tcr ORF (Myc-Tcr) (Fig. 1A). The construct $\left[\mathrm{Tg}\left(\mathrm{Smok}^{*} \mathrm{Tcr}\right) 9-987 \mathrm{Bgh}\right.$, abbreviated $\mathrm{Tg}$ 9] was introduced into the germline of mice. To ensure that this construct is able to cause TRD-like endogenous Tcr, we tested transgenic males for the transmission rate of $T g 9$ in the presence of $T c d$ genes. $T g 9 / 0$ males were crossed to females carrying the partial $t$ haploytpe $t^{h 51} t^{h 18} \mathrm{com}-$ prising several of the Tcd loci, but not Tcr (Lyon 1984). Male offspring of the genotype $\operatorname{Tg} 9 / 0 ; t^{h 51} t^{h 18} /++$ were crossed to wild-type females, and their litters were tested for the transmission rate of $\mathrm{Tg} 9$. We found that $76.6 \%$ of their offspring inherited the $T g 9$ construct (Table 1). Deviation from the Mendelian ratio was highly significant, demonstrating that $T g 9$ expresses Tcr function comparable with endogenous Tcr (Lyon 1984). On the 
A

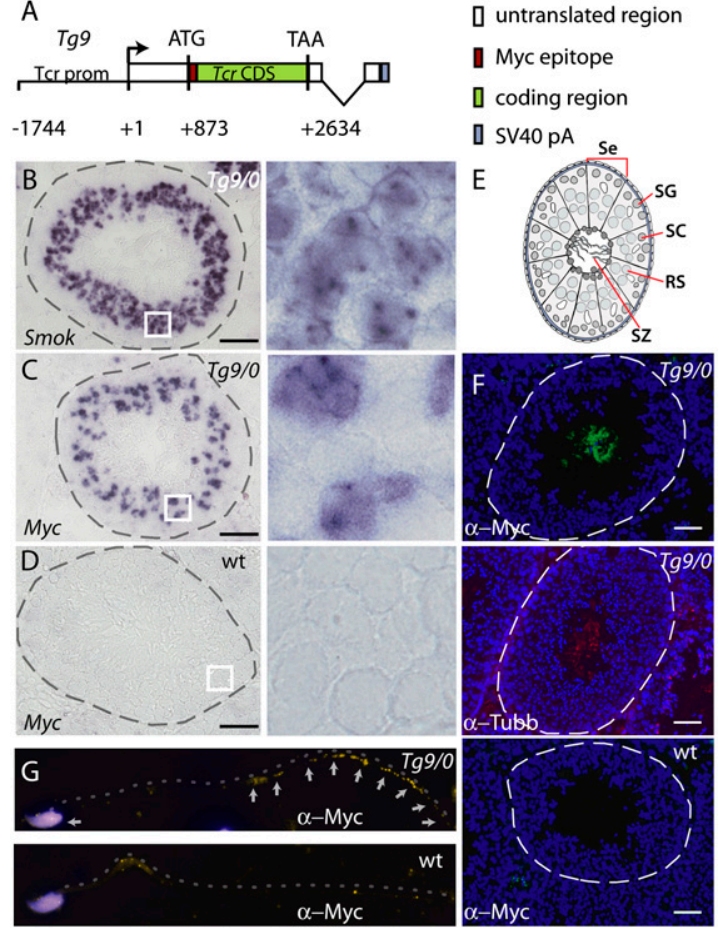

Figure 1. Tcr transcripts escape the general mechanism of product sharing between syncytial sperm cells and are translated at late stages of spermiogenesis. (A) Schematic drawing of the transgene construct $T g 9$ comprising the Tcr promoter (Tcr prom), untranslated region, and Myc epitope-tagged Tcr coding region (Tcr CDS), followed by the simian virus polyadenylation signal (SV40pA). $(B-D)$ Cryosections of $T g 9 / 0(B, C)$ or wild-type $(D)$ testes hybridized in situ with digoxygenin (DIG)-labeled Smok1-specific $(B)$ or Myc epitopespecific $(C, D)$ probes, showing expression of endogenous Smok1 transcripts in all round spermatids $(B)$, whereas $T g 9$ transcripts are confined to a subpopulation of round spermatids $(C)$ and not detected in the control $(D)$. Dashed lines indicate the outlines of seminiferous tubules, and white boxes indicate subregions shown at higher magnification on the right. Bar, $100 \mu \mathrm{m}$. (E) Schematic view of a seminiferous tubule. (Se) Sertoli cells; (SG) spermatogonia; (SC) spermatocytes; (RS) round spermatids; (SZ) spermatozoa. (F, top panel) Immunofluorescent detection of Myc-Tcr protein using an anti-Myc antibody $(\alpha-\mathrm{Myc})$ on testis cryosections of a $T g 9 / 0$ male reveals Tcr protein in the lumen of seminiferous tubules. (Middle panel) $\beta$-Tubulin $(\alpha$-Tubb), a major component of axonemes, indicating flagella, localizes to the same region. $(G)$ In epididymal spermatozoa, Myc-Tcr localizes primarily to the principal piece and nucleus (yellow arrows; the flagellum is indicated by a dashed line). (F,G, bottom panel) Wild-type controls show some nonspecific primary antibody reaction. Nuclei are visualized by DAPI staining (blue). Bar, $50 \mu \mathrm{m}$.

wild-type background, in the absence of Tcd activity, the transgene had no statistically significant effect.

RNA expression of the transgene construct was analyzed by in situ hybridization of cryosections derived from testes of adult hemizygous males (Fig. 1B,C). To distinguish $\mathrm{Tg}$ 9-derived transcripts from endogenous Smok1 expression, we used a probe specific for the Myc epitope tag of the transgene (Fig. 1C). Expression of wild-type Smok1 was analyzed on consecutive sections hybridized with a Smok1-specific probe (Fig. 1B). Both probes produced signals in a broad ring of cells located between the outer basal lamina and the lumen of seminiferous tubules, representing haploid round spermatids (Russell et al. 1990). Thus, the Tcr promoter used for the Tg9 construct confers the same spatial and temporal expression pattern as the endogenous Smok1 promoter, suggesting that it reflects the activity of the endogenous Tcr gene promoter (Herrmann et al. 1999). Our data showed that the RNA expression of Smok1 and Tcr is confined to haploid spermatids (Fig. 1B,C,E; Supplemental Fig. 2).

$T c r$ expression from the $T g 9$ transgene construct was analyzed in hemizygous males, and thus transcription of Tg9 is expected to take place only in the cells that obtained the transgene during meiosis-approximately half of the haploid round spermatids (Braun et al. 1989). Yet, if the RNA products were distributed throughout the syncytium, the Tg9-derived transcripts should be detectable in all round spermatids. However, we detected $\mathrm{Tg} 9$ derived RNA in only approximately half of the round spermatids. Therefore, our data suggest that Tcr transcripts escape the general mechanism of gene product sharing between neighboring cells of the syncytium (Braun et al. 1989) and remain, perhaps tethered, in the cells of origin (Fig. 1C). Indeed, high-resolution imaging showed that Smok1 as well as Tg9-derived Tcr transcripts occur mostly in RNA aggregates located inside or attached to the nucleus, consistent with retention of the RNA close to the site of production (Fig. 2).

RNA tethering, however, is not sufficient for haploid restriction of Tcr activity (Braun et al. 1989). Translational repression would also be required to prevent distribution of the protein between gametes. Therefore, we asked at what stage of spermatogenesis Tcr transcripts are translated. Testis sections derived from $\mathrm{Tg} 9 / 0$ males were immunostained with anti-Myc antibody to visualize Myc-Tcr protein by immunofluorescence. We observed Myc-Tcr in only the luminal region of seminiferous tubules of testes derived from $T g 9$ carrier males, but not in wild-type controls (Fig. 1F; Supplemental Fig. 3). The luminal aspect of seminiferous tubules harbors sperm cells reaching the end of differentiation as well as mature spermatozoa, which are released for transport to the epididymes (Russell et al. 1990). Much of the lumen is filled with flagella, as visualized by immunofluorescent detection of $\beta$-tubulin, a major component of the axoneme. High-resolution imaging of immunostained spermatozoa derived from the cauda epididymis revealed Myc-Tcr protein in the principal piece of flagella and in sperm nuclei (Fig. 1G). Thus, we conclude that Myc-Tcr protein is translated in flagellated spermatids and localized in the principal piece and nucleus of sperm.

The protein expression pattern of Tcr revealed an unexpected substantial delay between the onset of transcription in round spermatids and the appearance of protein in the flagella of late spermatids. These findings suggest high stability concomitant with strong translational repression of the Tcr transcripts throughout spermiogenesis. Although post-transcriptional control and translational repression are quite common for spermatogenic genes (Braun 1998; Hecht 1998), to our knowledge, such a late onset of protein expression during spermiogenesis has not yet been reported.

Immunofluorescent detection of Myc-Ter on isolated spermatozoa did not allow for analysis of Tcr distribution, namely, if between meiotic partners or if retained in the cells of origin. To analyze this, we used high-resolution electron microscopy techniques to detect gold-labeled antiMyc immunostaining of Myc-Tcr on ultrathin sections of spermatozoa contained in the epididymis of $\mathrm{Tg} 9 / 0$ males. 
Table 1. The transgene construct $\mathrm{Tg} 9$ causes non-Mendelian inheritance in the presence of $\mathrm{Tcd} s$

\begin{tabular}{lccccccc}
\hline & & \multicolumn{5}{c}{ Offspring } \\
\cline { 3 - 8 } Genotype of male & Number of males & $T g$ & Non- $T g$ & Total & Percent $T g$ & $\chi^{2}$ & $P$-value \\
\hline$T g 9 / 0 ; t^{h 51} t^{h 18} /++$ & 3 & 143 & 39 & 182 & $76.6 \%$ & 59.4 & $1.27 \times 10^{-14 a}$ \\
$T g 9 / 0 ;++/++$ & 3 & 141 & 113 & 254 & $55.5 \%$ & 3.09 & $0.0789^{\mathrm{b}}$ \\
\hline
\end{tabular}

$\chi^{2}$ method was used.

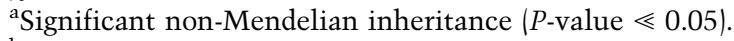

${ }^{\mathrm{b}}$ Nonsignificant deviation from Mendelian inheritance $(P$-value $>0.05)$.

Gold particles were detected in sperm nuclei and in flagella of epididymal spermatozoa (Fig. 3). Gold particles observed in the flagella localized to outer dense fibers (ODFs) and at the transversal ribs of the fibrous sheath (Fig. 3A), consistent with a putative role of Tcr in flagellar motility. These flagellar structures are known to anchor components of Rho signaling pathways (Fujita et al. 2000; Eddy et al. 2003), further supporting a functional link between Tcr and Rho signaling, as revealed previously by genetic data (Bauer et al. 2005, 2007). The significance of Tcr protein in the sperm nucleus remains unclear.

Immunogold particles were counted from 204 and 202 sections of sperm nuclei derived from the epididymis of $\mathrm{Tg} 9 / 0$ and wild-type control males, respectively. In the control sample (wild type), about two-thirds of the sperm nuclei were devoid of immunogold particles; however, the remainder showed low counts of false-positive staining (up to four particles per sperm nucleus). In contrast, Tg9/0-derived sperm heads showed considerably higher gold particle counts (up to 22 particles) in two-thirds of the sections, whereas about one-third of the sections contained no particles at all (Fig. 3C). Statistical analysis revealed the nonhomogeneous distribution of particle counts in $T g 9 / 0$-derived sperm was due to a significant subpopulation of sperm being wild type with respect to Myc-Tcr protein. Maximum likelihood estimation indicated that this subpopulation amounts to about half of the sperm (Supplemental Material). Therefore, consistent with previous observations (Fig. 1C) we conclude that Tcr protein is retained in the haploid sperm cells expressing the gene.

Our data provide a plausible molecular explanation for phenotypic inequality of sperm derived from $t /+$ males, leading to TRD. We show that Tcr is post-meiotically transcribed in haploid spermatids, and the mRNA is translationally repressed and tethered, preventing transport to neighboring cells. Delayed translation of the mRNA just prior to, or after, individualization of spermatozoa appears to prevent distribution of the protein between the gametes. Alternatively, translation of the mRNA may occur concomitant with the formation of flagella, and the Tcr protein might be specifically transported to the principal piece (Kierszenbaum 2002) and anchored at the fibrous sheath and ODFs.

This is strikingly in contrast to Tcds, as exemplified by Tagap1 and Fgd2, which are expressed premeiotically in diploid spermatocytes (Bauer et al. 2005, 2007). Their gene products are expressed in all cells of the syncytium and compromise the flagellar function of all spermatozoa derived from a $t /+$ male (Bauer et al. 2005, 2007). Due to haploid restriction of Tcr gene products, which have rescuing activity, only $t$ sperm are rescued from the deleterious effect of the Tcds. Thus, + sperm remain defective and $t$ sperm gain an advantage in reaching and fertilizing egg cells. In that manner, it is apparent that Tcr takes advantage of the $T c d$ function and selfishly uses its special properties to promote its own transmission to the next generation. However, since the Tcds and Tcr are locked in a linkage group protected from splitting up by recombination suppression (Lyon 1984), the Tcd genes may also profit from the enhanced transmission rate of Tcr.

Since Tcr is a mutated gene, we questioned whether the tethering of transcripts and late translation of Tcr transcripts, which enables haploid restriction of Tcr function, are due to neomorphic mutations, and thus are unique to $T c r$ or, alternatively, exist also in wild-type Smok1, the progenitor of $T c r$. If the latter were true, it would suggest that similar properties also exist in other genes not associated with $t$ haplotype-dependent TRD, since it is

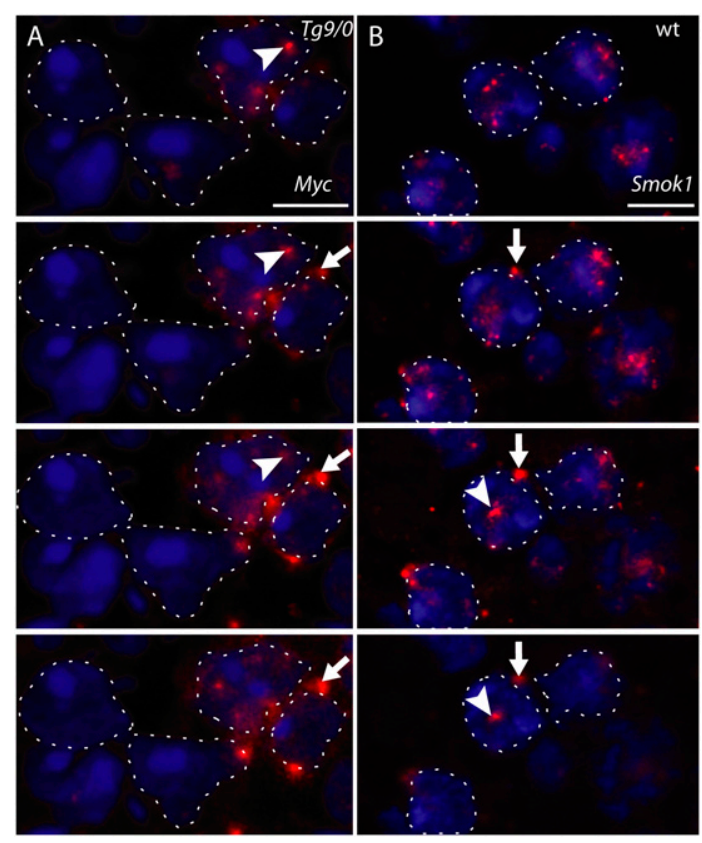

Figure 2. Tcr and Smok1 transcripts occur mainly in nuclear and perinuclear aggregates. Serial optical sections $(\sim 1-\mu \mathrm{m}$ depth) of testicular cryosections obtained by confocal microscopy visualizing Tg9 $(A)$ or Smok1 $(B)$ transcripts (red) detected by fluorescent in situ hybridization in round spermatids. Selected examples of nuclear (arrowhead) and perinuclear (arrow) RNA aggregates are indicated. $\mathrm{Tg} 9$ transcripts were detected on sections of $\mathrm{Tg} 9 / 0$-derived testes (shown in A) with a DIG-labeled Myc epitope-specific probe, and Smok1 transcripts were detected on sections of wild-type testes with a Smok1-specific probe. Dashed lines denote the nuclear outlines of round spermatids visualized by DAPI staining (blue), and bright blue structures within the nuclei represent nucleoli. Bar, $6.5 \mu \mathrm{m}$. 


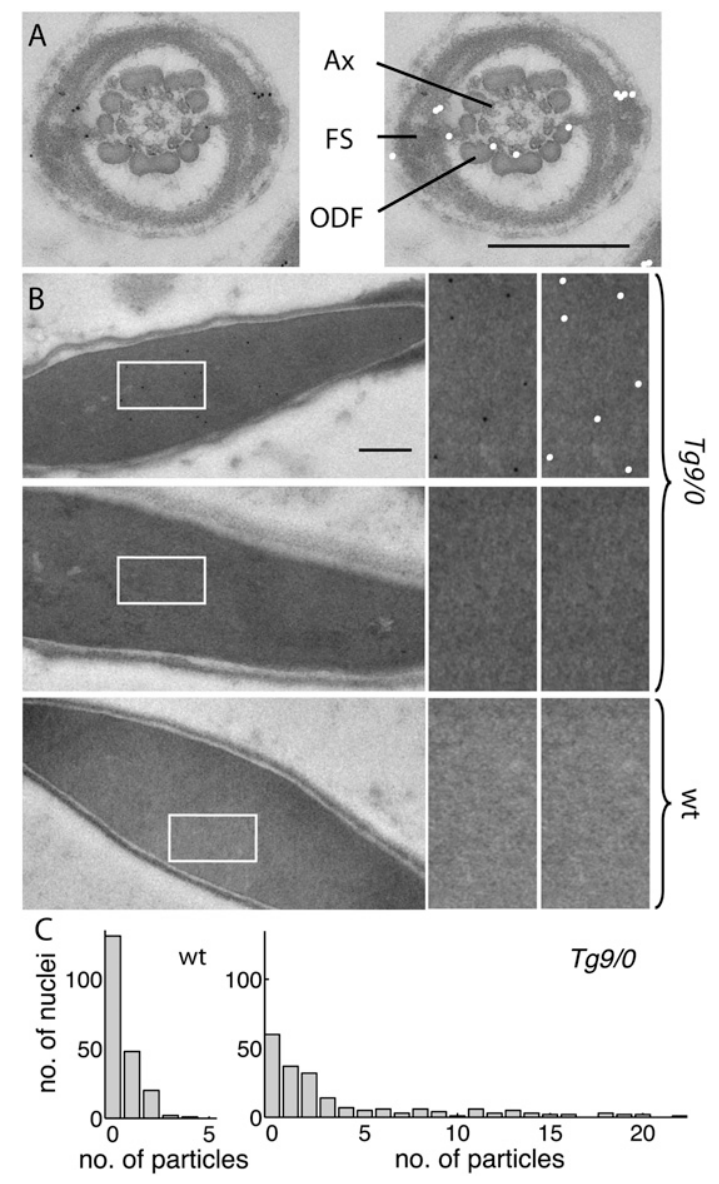

Figure 3. Tcr protein is retained in the spermatozoa expressing the transgene. $(A, B)$ Ultrathin sections of spermatozoa derived from the cauda epididymis of $T g 9 / 0$ or wild-type control (wt) males assayed for Myc-Tcr protein using immunogold-labeled anti-Myc antibodies reveals the localization of Tcr protein at the fibrous sheath (FS) and the ODFs of the principal piece of the flagellum $(A)$ and in sperm nuclei $(B)$. A fraction of $T g 9 / 0$-derived sperm nuclei show no or few immunogold particles, like wild-type sperm. White boxes indicate areas shown at higher magnification on the right ; for better visualization, immunogold particles are shown as white spots on duplicate panels. (Ax) Axoneme. Bars: $A, 500 \mathrm{~nm} ; B, 280 \mathrm{~nm}$. (C) Histograms showing the number of sections of sperm nuclei corresponding to the number of immunogold particles counted, based on the analysis of 202 and 204 sperm nuclei derived from wild-type (wt) or Tg9/0 animals, respectively. In sperm nuclei of hemizygous $T g 9 / 0$ males, one part of the distribution is concentrated at low immunogold particle counts, whereas the remainder is widely spread at higher counts (heavy tail distribution).

unlikely that such a refined mechanism of post-transcriptional regulation in haploid spermatids is unique to a single gene family. To answer this question, we investigated the properties of Smok1 expressed from a transgene construct in hemizygous males. A minigene for wild-type Smok1 $(\mathrm{TgS})$ was constructed and introduced into the germline of mice (Fig. 4A). Males carrying $\mathrm{TgS}$ were tested for RNA and protein expression of the transgene construct, similar to $T g 9$. Our results show that the RNA and protein expression patterns of $T g S$ are indistinguishable from those obtained with $\mathrm{Tg} 9$; i.e., restricted RNA expression in round haploid spermatids, tethering of the transcripts in the cells of origin, and late translation of the protein in flagellated spermatids (Fig. 4B,C).
These data suggest that the post-transcriptional control mechanisms revealed for Tcr and Smok1 indeed existed prior to the evolution of Tcr from Smok1. Thus, this mechanism is most likely not rare in genes involved in sperm function, but simply has not been noticed before. It is conceivable that genes that are involved in sperm function, and whose products escape the sharing mechanism between spermatids (Martin-DeLeon et al. 2005), have the potential to evolve functionally different alleles, which may cause phenotypic inequality of gametes and non-Mendelian inheritance. We propose that the molecular mechanism regulating Tcr/Smok1 elucidated here may be a common cause of non-Mendelian inheritance in mammals (Fig. 4D). It may also extend to humans, although it is unclear whether any of the non-Mendelian inheritance phenotypes described in humans thus far (Curtsinger et al. 1983; Van Heyningen and Yeyati 2004; Hesketh and Xing 2006; James 2009) relate to the mechanism shown here. Our data describing the regulation of $\mathrm{Tcr} / \mathrm{Smok} 1$ provide a molecular basis for the identification of other genes that may be involved in non-Mendelian inheritance. This will promote the

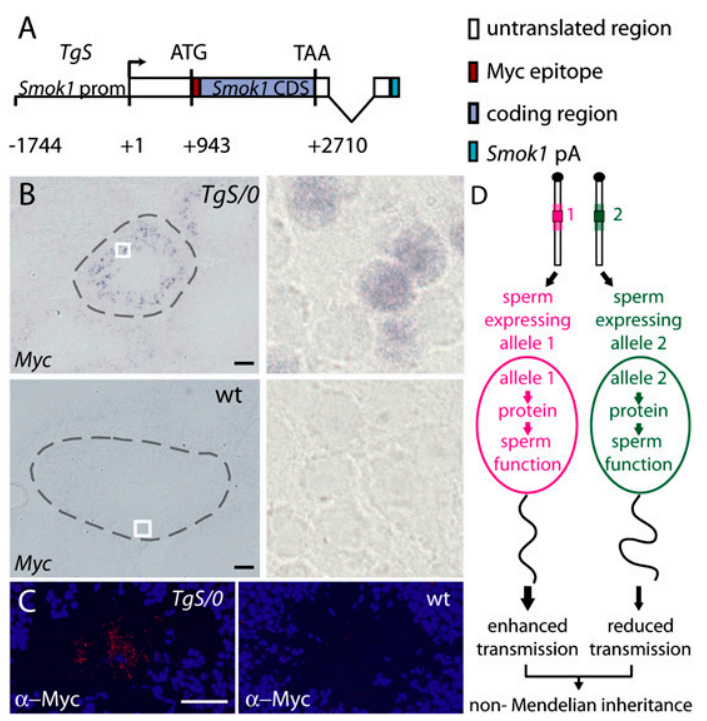

Figure 4. Smok1 shows the same pattern of transcriptional expression and post-transciptional control as Tcr. (A) Schematic drawing of the transgene construct $\mathrm{TgS}$, consisting of the Smok1 promoter (Smok1 prom), the Myc epitope-tagged Smok1 coding region (Smok1 CDS), and untranslated regions followed by the polyadenylation signal (Smok1pA). (B) Testis cryosections of TgS/O (top panel) or wild-type control (bottom panel) males hybridized in situ with a DIGlabeled Myc epitope-specific probe (Myc) show $\mathrm{TgS}$ expression in seminiferous tubules of the $T g S / 0$ male in a subpopulation of round spermatids, but not in the control. White boxes indicate the regions shown at higher magnification on the right, and the borders of seminiferous tubules are indicated by dashed lines. Bar, $100 \mu \mathrm{m} .(C)$ Immunofluorescent detection of Myc-Smok1 protein on cryosections of seminiferous tubules derived from a $\mathrm{TgS} / 0$ (left panel) or a wildtype control (right panel) male using anti-Myc antibodies visualizing Myc-Smok1 protein (red) specifically in the lumen filled with flagella of late-stage spermatids. Nuclei are visualized by DAPI staining (blue). Bar, $50 \mu \mathrm{m}$. (D) Model depicting a mechanism leading to non-Mendelian inheritance of functionally different alleles (denoted 1 and 2), as well as genetically linked chromosomal regions (shaded area), of a gene involved in sperm function due to post-meiotic expression and retention of the gene products in the haploid spermatids, thus creating functionally different spermatozoa. 
investigation of this striking phenomenon in humans and other organisms.

\section{Materials and methods}

\section{Generation of transgenic mice}

The $T c r$ transgene construct $T g\left(S_{m o k}{ }^{*} T c r\right) 9-987 B g h(T g 9)$ consists of the $T c r$ promoter (1744 base pairs [bp] upstream of the transcriptional start site) and the Tcr 5 - -untranslated region (UTR) amplified from testis cDNA, followed by six repeats $(6 \times)$ of the Myc epitope fused $\mathrm{N}$-terminally to the coding sequence of $T c r^{t w 12}$. The coding sequence and part of the $3^{\prime}$-UTR and intron 2 were obtained from $t^{w 12}$ genomic DNA. In order to eliminate residual kinase acivity of Tcr, we introduced the mutations D127A and D131A in subdomain VIb. To avoid the large Rsk3-derived 3'-UTR of Tcr, part of intron 2 and $141 \mathrm{bp}$ of the third exon encoding $3^{\prime}$-untranslated sequences were derived from genomic DNA of $S m o k 1^{\text {tw12 }}$. The remainder of the $3^{\prime}-$ UTR and the polyadenylation signal of $T g 9$ are derived from SV40 DNA.

For generation of the wild-type Smok1 transgene $\mathrm{Tg}(\mathrm{Smok} 1) 1-8 \mathrm{Bgh}$ $(T g S)$, we amplified the Smok1 promoter and the $5^{\prime}$ end of the Smok1 5'-UTR from C57Bl/6J genomic DNA. To exclude intron 1 of the Smok1 gene, we amplified the $3^{\prime}$ end of the $5^{\prime}$-UTR sequence from testis cDNA (C57Bl/6J). The Smok1 coding region, its 3'-UTR, intron 2, and polyadenylation signal were amplified from genomic DNA. The $\mathrm{N}$ terminus of the coding sequence was fused in-frame to a $6 \times$ Myc epitope tag, as for $T g 9$.

Transgenic constructs were isolated from the vector backbone and purified for pronuclear injection of fertilized C57Bl/6 eggs using standard procedures.

GenBank accession numbers are AJ245456 for Tcr AJ245452 and AJ245455 for Smok1 ${ }^{\text {C57BL/6 }}$; Smok ${ }^{t w 12}$.

\section{TRD tests}

We backcrossed $T g 9$ founders for at least four generations to the strain $\mathrm{BTBR} / \mathrm{TF}-\mathrm{tf} / \mathrm{ttf}$, our preferred strain for testing TRD (Gummere et al. 1986). We mated backcrossed $T g 9 / 0$ animals to the partial $t$ haplotype $t^{h 51} t^{h 18} /++(B T B R / T F)$ carrying Tcd1 and Tcd2, but not Tcr (Lyon 1984).

To determine the transmission rate of the $T g 9$ transgene in the presence and absence of $T c d s$, we mated $T g 9 / 0 ; t^{h 51} t^{h 18} /++(B T B R / T F)$ tester males and $T g 9 / 0 ;++/++(\mathrm{BTBR} / \mathrm{TF})$ control males with outbred females (NMRI). Embryos were dissected at 9-14 d post-coitum and lysed using standard procedures. After dilution of the lysate in water $(1: 20)$ and heat inactivation $\left(20 \mathrm{~min}, 80^{\circ} \mathrm{C}\right)$, genotyping was performed by PCR using primers NV55 and NV34 (Supplemental Material).

To statistically analyze whether the transmission ratio of the transgene significantly deviates from the Mendelian ratio, we performed a $\chi^{2}$ test (Tg9/0;++/++: $n=254, \alpha=0.05$; and Tg9/0; $\left.t^{h 51} t^{h 18} /++: n=182, \alpha=0.05\right)$.

\section{Transcript analysis}

In situ hybridization was performed on $10-\mu \mathrm{m}$ testis cryostat sections essentially as described (Brent et al. 2003). We detected transgenic transcripts from $T g 9$ and $T g S$ using a digoxygenin (DIG)-labeled riboprobe hybridizing to the $6 \times$ Myc epitope tag. To generate the template for probe synthesis, we subcloned a PCR fragment (primers NV33 and NV34; Supplemental Material) containing the $6 \times$ Myc sequence amplified from $\mathrm{Tg} 9$ into pCRII-TOPO (Invitrogen) and obtained riboprobes by antisense in vitro transcription from this vector. For the detection of endogenous wildtype Smok1 transcripts, we PCR-amplified $1.3 \mathrm{~kb}$ of the protein-coding region of Smok1 (129Sv) from a 2.2-kb genomic EcoRI fragment of the Smok1 gene in pBS-KS (primers BGH170 and NV19; Supplemental Material). We subcloned the PCR fragment in pCRII-TOPO and used it as a template for in vitro transcription of a DIG-labeled antisense riboprobe. For the development of the fluorescent in situ hybridization signal, we used Fast Red Substrate (DAKO), as recommended by the supplier. Nuclei were counterstained with DAPI.

\section{Immunofluorescence detection of protein on testis sections and epididymal sperm}

We performed immunofluorescence detection of protein on testis cryostat sections after pretreatment of the tissue as described (Brent et al. 2003).
Testis sections of $T g 9 / 0$ and BTBR/TF control animals were blocked with PBS, $0.2 \%$ Tween (PBS-T) and 5\% normal goat serum (Vector Laboratories), and incubated with a mouse monoclonal anti-Myc antibody (1:100; clone 4A6, Upstate Biotechnologies) or a rabbit polyclonal antiMyc antibody (1:100; Sigma, C3956) and, subsequently, with a peroxidasecoupled sheep anti-mouse or a donkey anti-rabbit IgG (both from Amersham Biosciences), respectively. Signals were amplified by FITC-coupled TSA (Perkin Elmer) substrate reaction as recommended by the supplier. For $\beta$-tubulin staining on $T g 9 / 0$ testis sections, we used a mouse monoclonal anti- $\beta$-tubulin antibody from ascites fluid (1:100; Sigma, T5293) and a Cy3-coupled goat anti-mouse IgG (Jackson ImmunoResearch).

Immunofluorescence on testis sections of transgenic $\mathrm{TgS} / 0$ and BTBR/ TF control animals was performed by blocking with PBS-T and $5 \%$ normal goat serum, and incubation with a mouse monoclonal anti-Myc antibody (1:100; clone 4A6, Upstate Biotechnologies) and, subsequently, with a Cy3-coupled goat anti-mouse IgG . Nuclei were counterstained with DAPI.

For the visualization of Tcr protein in sperm, mature spermatozoa were isolated from the cauda epididymis and pretreated as described (Braun et al. 1989). Briefly, sperm was capacitated, washed, spread on glass slides, and fixed with $4 \%$ PFA/PBS. After a blocking step with $5 \%$ normal goat serum in PBS-T, we incubated fixed spermatozoa with a rabbit polyclonal anti-Myc antibody (1:100; Sigma C3956) and Qdot 655-conjugated goat anti-rabbit IgG (Invitrogen). Sperm nuclei were counterstained with DAPI. Qdot 655 fluorescence was monitored using a suitable filter set (Chroma Technology).

\section{Electron microscopy of sperm}

Cauda epididymis containing mature spermatozoa was isolated from $\mathrm{Tg} / / 0$ males and from BTBR/TF control animals, dissected in fixative (glutaraldehyde, PFA), and fixed overnight at $4^{\circ} \mathrm{C}$. Tissues were dehydrated in an ethanol series, transferred to LR white resin (Electron Microscopy Sciences), polymerized for $72 \mathrm{~h}$ at $42^{\circ} \mathrm{C}$, and subsequently cut into $70-\mathrm{nm}$ ultrathin sections. Immunogold labeling was performed with a rabbit polyclonal anti-Myc antibody (Sigma) and $10 \mathrm{~nm}$ of gold-conjugated anti-rabbit IgG (British Biocell) and documented by electron microscopy on a CCD-camera (FastScan, TVIPS).

Sperm nuclei areas and respective gold particle counts were subsequently analyzed on digital pictures (ImageJ software) and statistically analyzed as described below.

\section{Statistical analysis of immunogold particle counts}

To analyze the distribution of Tcr protein among sperm from $\mathrm{Tg} 9 / 0$ hemizygous mice, we assumed that the particle counts obtained from immunogold labeling are Poisson distributed, which follows if binding events occur independently of each other with a constant rate (Gardiner 2004; Ewens and Grant 2005). Using sperm from wild-type mice, we studied the false-positive signal caused by the labeling technique. Based on this estimate, and considering the number of sperm that showed no particles, we carried out a binomial test $(n=51, \alpha=0.01)$ to analyze whether, in $T g 9 / 0$ -derived sperm, Tcr is distributed to all sperm or whether it remains restricted to a subpopulation of sperm, and applied maximum likelihood estimation to estimate the proportion of the subpopulation devoid of Tcr protein (Supplemental Material).

\section{Acknowledgments}

We thank Rudi Lurz for electron microscopy, M.F. Lyon for mice carrying the $t$ haplotypes used in this study, Vijay Subramanian for suggestions and discussions about statistical approaches, Ingo Voigt for pronuclear injection, Ludger Hartmann for supervision of the animal facility, Carolin Willke for expert animal caretaking, and Alexandra Farrall and Phillip Grote for critical comments on the manuscript. This project was supported by a grant from the Deutsche Forschungsgemeinschaft to B.G.H.

\section{References}

Bauer H, Willert J, Koschorz B, Herrmann BG. 2005. The t complexencoded GTPase-activating protein tagap1 acts as a transmission ratio distorter in mice. Nat Genet 37: 969-973. 


\section{Véron et al.}

Bauer H, Veron N, Willert J, Herrmann BG. 2007. The t-complex-encoded guanine nucleotide exchange factor Fgd2 reveals that two opposing signaling pathways promote transmission ratio distortion in the mouse. Genes \& Dev 21: 143-147.

Braun RE. 1998. Post-transcriptional control of gene expression during spermatogenesis. Semin Cell Dev Biol 9: 483-489.

Braun RE, Behringer RR, Peschon JJ, Brinster RL, Palmiter RD. 1989. Genetically haploid spermatids are phenotypically diploid. Nature 337: 373-376.

Brent AE, Schweitzer R, Tabin CJ. 2003. A somitic compartment of tendon progenitors. Cell 113: 235-248.

Chesley P, Dunn LC. 1936. The inheritance of taillessness (anury) in the house mouse. Genetics 21: 525-536.

Curtsinger J, Ito R, Hiraizumi Y, Curtsinger JW. 1983. A two-generation study of human sex-ratio variation. Am J Hum Genet 35: 951-961.

Eddy EM, Toshimori K, O'Brien DA. 2003. Fibrous sheath of mammalian spermatozoa. Microsc Res Tech 61: 103-115.

Ewens WJ, Grant G. 2005. Statistical methods in bioinformatics Springer, New York.

Fujita A, Nakamura K, Kato T, Watanabe N, Ishizaki T, Kimura $\mathrm{K}$ Mizoguchi A, Narumiya S. 2000. Ropporin, a sperm-specific binding protein of rhophilin, that is localized in the fibrous sheath of sperm flagella. J Cell Sci 113: 103-112.

Gardiner CW. 2004. Handbook of stochastic methods: For physics, chemistry and the natural sciences. Springer, New York.

Gummere G, McCormick P, Bennett D. 1986. The influence of genetic background and the homologous chromosome 17 on t-haplotype transmission ratio distortion in mice. Genetics 114: 235-245.

Hecht NB. 1998. Molecular mechanisms of male germ cell differentiation. Bioessays 20: $555-561$

Herrmann B, Koschorz B, Wertz K, McLaughlin K, Kispert A. 1999. A protein kinase encoded by the $t$ complex responder gene causes nonmendelian inheritance. Nature 402: 141-146.

Hesketh T, Xing ZW. 2006. Abnormal sex ratios in human populations: Causes and consequences. Proc Natl Acad Sci 103: 13271-13275.

Hurst GD, Werren JH. 2001. The role of selfish genetic elements in eukaryotic evolution. Nat Rev Genet 2: 597-606.

James WH. 2009. The variations of human sex ratio at birth during and after wars, and their potential explanations. I Theor Biol 257: 116123.

Katz D, Erickson R, Nathanson M. 1979. Beat frequency is bimodally distributed in spermatozoa from t/t12 mice. J Exp Zool 210: 529-535.

Kierszenbaum AL. 2002. Intramanchette transport (imt): Managing the making of the spermatid head, centrosome, and tail. Mol Reprod Dev 63: $1-4$.

Lyon MF. 1984. Transmission ratio distortion in mouse t-haplotypes is due to multiple distorter genes acting on a responder locus. Cell 37: 621-628

Lyon MF. 2003. Transmission ratio distortion in mice. Annu Rev Genet 37: 393-408

Martin-DeLeon PA, Zhang H, Morales CR, Zhao Y, Rulon M, Barnoski BL, Chen H, Galileo DS. 2005. Spam1-associated transmission ratio distortion in mice: Elucidating the mechanism. Reprod Biol Endocrinol 3: 32 . doi: 10.1186/1477-7827-3-32.

Olds-Clarke P, Johnson LR. 1993. T haplotypes in the mouse compromise sperm flagellar function. Dev Biol 155: 14-25.

Russell L, Ettlin R, Hikim A, Clegg E. 1990. Histological and histopathological evaluation of the testis. Cache River Press, Saint Louis, MO.

Schimenti J. 2000. Segregation distortion of mouse $t$ haplotypes the molecular basis emerges. Trends Genet 16: 240-243.

Van Heyningen V, Yeyati PL. 2004. Mechanisms of non-Mendelian inheritance in genetic disease. Hum Mol Genet 13: R225-R233. doi $10.1093 / \mathrm{hmg} / \mathrm{ddh} 254$.

Ventela S, Toppari J, Parvinen M. 2003. Intercellular organelle traffic through cytoplasmic bridges in early spermatids of the rat: Mechanisms of haploid gene product sharing. Mol Biol Cell 14: 2768-2780. 


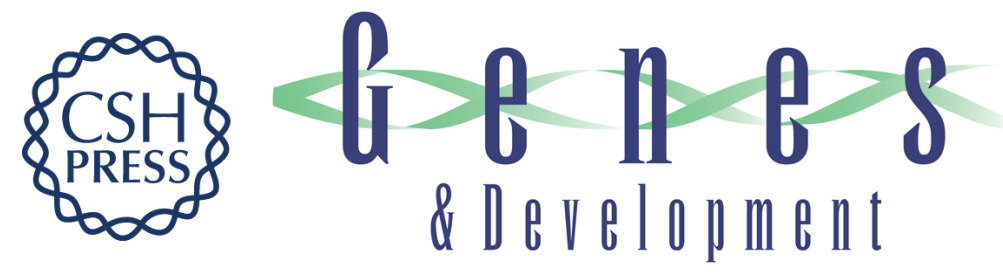

\section{Retention of gene products in syncytial spermatids promotes non-Mendelian inheritance as revealed by the $t$ complex responder}

Nathalie Véron, Hermann Bauer, Andrea Y. Weiße, et al.

Genes Dev. 2009, 23:

Access the most recent version at doi:10.1101/gad.553009

Supplemental http://genesdev.cshlp.org/content/suppl/2009/11/30/23.23.2705.DC1
Material

References This article cites 24 articles, 4 of which can be accessed free at:

http://genesdev.cshlp.org/content/23/23/2705.full.html\#ref-list-1

License

Email Alerting Receive free email alerts when new articles cite this article - sign up in the box at the top

Service

right corner of the article or click here.

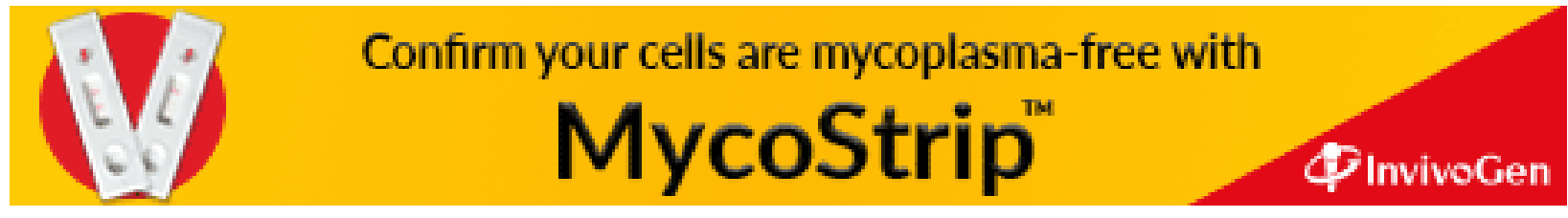

